IV. Aus der medic. Abtheilung des Bürgerhospitales zu $\mathrm{Köln} \cdot \mathrm{a} . \mathrm{Rh}$.

\section{Zwei Fälle von Compression des Rückenmarkes durch Wirbelkrebs.}

Voll

\section{Dr. Heinrich Simon.}

\author{
(Fortsetzung aus No. 23.)
}

Ich stelle noch in Kurzem aus der Krankengeschichte den Tem peraturverlauf vom 1 . April an zusammen.

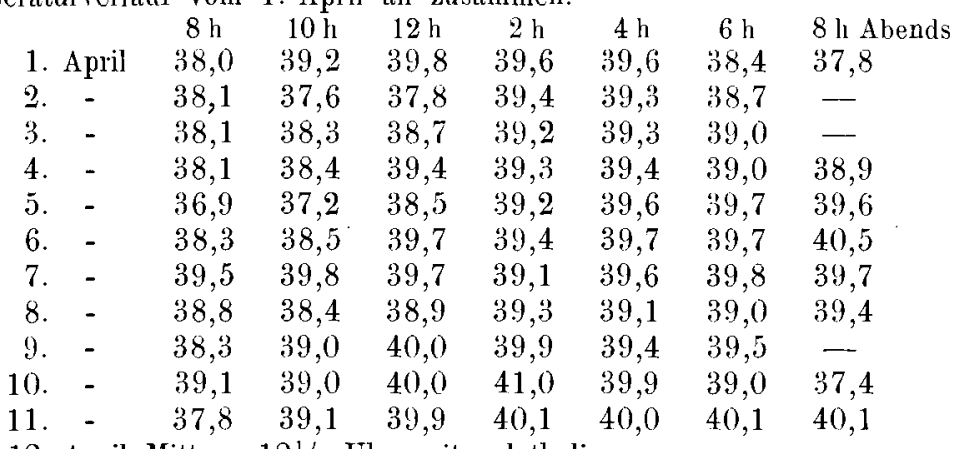

12. April Mittags 12\%. Uhr exitus lethalis.

Die Section wurde am 13. April Vormittags 11 Uhr von Herrn Prof. Dr. Leichtenstern vorgenommen.

"Mittelgrosse, weibliche Leiche von allgemeiner Abmagerung, schmutzig weisser, schlaffer Haut, dürftiger Musculatur. Spärliche blasse Todtenflecke, geringe Leichenstarre.

An der Rückelffäche des Rumpfes eill die ganze Kireuzbeingegend und den grössten Theil der Nates einnehmendes, missfarbiges, gangranöses Decubitus-Geschwür, dessen Zerstörung bis an das Os sacrum heranreicht. Ceber beiden Trochanteren kreisrunde, thalergrosse, mit schwarzen, blutigen Krusten bedeckite I)ecubitus-Geschwüre.

Das Schädeldach symmetrisch mehr brachycephal, dünn. Die Diploë blass, blutann.

Die Dura mater schlaff, zart, dünı, blutarm. Längsblutleiter fast bhitleer.

Die Flüssigkeit im Arachıoidealraum vermehrt, die weichen Häute über ver Convexität blutarm, über Scheitel und Hinterhauptshirn ädematös infiltrirt und schwach milchig getrübt.

Gehirmoberfläche und Basis ohne besondere Anomalie. Das Gehirn ausserordentlich blutarm, stark durchfeuchtet, schneidet sich zähe. Ventrikelflüssigkeit vermindert, Ventrikel enge.

Sonst ansser allgemeiner Anämie und stärkerer seröser Durchfeuchtung nichts Abnornies zu erkennen."

Ich übergehe den Sectionsbefund der Brustorgane, da er ausser anämischer und ölematöser Beschaffenheit der Luugen, geringfügigen Transsudat in heiden Pleuraräumen, Schlaffheit des Herzens, geringem Gehalt der Herzhöhleu an Cruor und Faserstoffgerimuselı, keine nus näher interessirenden Thatsachen enthält.

In der Bauchhöhle ca. $250 \mathrm{ccm}$. klareı, gelben Serums. Die Lage der Bauchorgane die normale. Das Netz fettarm, dünn, dessen Venen stärker gefüllt. Das Gekrïse fettarm. Die Darmschlingen blass, blutarm, desgleichen das Peritoneum parietale. Der I) ünutarm mit Ausnahme weniger Strecken stark contrahirt, mit sehr geringen Inhalt, desgleichen starke Contraction des Colons. Magen klein, contrahirt. Die Schleimhaut des Tractus iutestinalis blass, vielfach mit zälıen Schlein bedeckt, ohıe besondere Anomalie.

Die Milz veröüssert, $16 \mathrm{~cm}$. lang, $11 \mathrm{~cm}$. breit, breiig, weich blau rüthlich braun. Die L eber beträchtlich vergrössert, $29 \mathrm{~cm}$. breit, $22 \mathrm{~cm}$. lang, 10 dick, schlaff, blutarm. Der acinüse Bau undeutlich. Die Farbe ziemlich gleichmässig grau-braun, trübe. Gallenblase wenig gefüllt, Galle gelbbraun, trübe. Pancreas klein, atrophisch.

Nach Entfernung der intraperitonealen Baucheingeweide präsentirt sich die quer vor der Wirbelsăule zur Cava inferior herüberziehende Vena renalis sinistra, als ein reichlich Q Querfinger breiter, dicker Wulst. Derselbe ist mit einem leicht zerbrockelnclen, weichen, speckigeu, graurothen Thrombus erfïllt, der sich zuspitzend eben etwas in das Lumen der Vena cava hereinragt. Desgleichell ist die aus der thrombotischen Vena renalis sinistra entspringende Vena spermatica interua in einen kleinfingerdicken, mit denselben grauröthlichen weichen Thrombusmassell erfüllten Strang verwandelt. Die Thrombose durchsetzt die ganze Vena spernatica interna von ihrer Eiumündung:sstelle in tie linke Vena renalis an bis zum Eintritt derselben in das breite Mutterband. Hier allgelangt setzt sich die Thrombusmasse in die Venen des Plexus pampiniformis des linken Eierstockes und des Ligam. uteri latum, sowie in conttinuo in die Venengeflechte der Scheide und urethra bis zu der Stelle fort, wo der bereits intra vitam (siehe oben lirankengeschichte) constatirte, als Thrombus vaginae diagnosticirte Tumor liegt, der sich auch als ein Thrombus erweist." ")

Es sei gleich hier aus der nachträglichen mikroskopischen Untersuchung eingefügt, dass es sich um eine krebsige Venen-Thrombose, unı Fortwucherung des Krebses der linken Niere in die Venta renalis und deren $Z_{w}$ wig, die Vena spermatica interna hinein handelt.

"Die linke Niere stellt einen voluminösen weichen, fluctuirenden Tumor dar von $22 \mathrm{~cm}$. Iä̈nge, $8 \mathrm{~cm}$. Breite und reichlich $8 \mathrm{~cm}$. Dicke. Dieser Tumor bewahrt die Gestalt der Niere. Seine überall glatte Oberfläche wird von der ausserordentlich verdünten, durchscheinenden $\mathrm{K}$ a ps el gebildet, durch welche flache gelbe Knoten durchschimmerı. Der Nierentumor bietet auf dem Durchschnitte ein farbenreiches, und auch sonst sehr differentes Bild dar. Die normale Structur der Niere, die Unterscheidung zwischen Rinde und Pyramide ist nur in einem ganz kleinen Bezirke eben noch erkemubar.

Die übrigen Abschnitte bilden weiche, gelbe, grüssere und kleinere, vielfach zusainmengeflossene, z.um Theil aher durch derhe Septa in .verschieden grosse Fïicher getrenute Knoten, welche vou gelher grau-röthlicher oder, ill Folge von Hämorrhagien, dunkelrother Farbe sind, bald etwas consistenter, bald im Centrum breiigweich und zerfliessend sind, auf letztere Weise ('ysten mit breiigem zerfallenem Inhalt darstellend. Das ganze Nierentecken ist mit diesen weichen, graugelben und graurothen IIassen erfiillt, welche sich auf eine Strecke von $8 \mathrm{~cm}$ in den linkel Ureter hinein fortsetzen, diesen vollständig obturirend."

Lis sei hier ans der späteren mikroskopischen [intersuchung angeführt, dass diesellue das Vorhandensein eines ächten Krebses der Niere ergab.

Wie rechte Niere ist etwas vergrïssert, $1: 3 \mathrm{~cm}$. ling, $6 \mathrm{~cm}$. breit, von normaler Dicke. Die Kapsel leicht und glatt abziehlar. Oberflache glatt, die Rinde etwas verhreitert, blass und trühe, sehr spärlich und ungleichmässig aber grol, roth gestrichelt, die Pyramiden blass blänlich. Dis ()rgan ist schlaff, weich, sehr blutarm.

Harnblase und Urethralschleimhaut hieten nichts Bemerkenswerthes.

Der Uterus ist schlaff, vergrüssert, $16 \mathrm{~cm}$. lang, am Fundus $10 \mathrm{~cm}$. breit. Seine Schleimhant ist dunkelbraunroth, an einzelnen Partien mit graugrin̈lu necrotischen Massen besetzt. (Oberflächliche SchleimlaantNecrose.)

1)ie Eierstücke ohne Anomalie.
Die Untersuchnng der Lymphdrüseu des Iesenteriums, der (ilandulae

Die Untersuchung der Lymphdrüseu des llesenteriums, der (xlandulae
paticae, coeliacae, sowie der retroperitonealen Irüsen ergiebt durchaus normale Beschaffenheit derselben.

Desgleichen ist die ummittelhare Umgebung der krelssigen linken Niere durchaus normal. Die Krebswithenng imnerhalb ter Niere hat an keiner Stelle deren Kiapsel durchbrochen.

Die vordere Flache der Wirbelkörper ohne jede Anomalie.

Bei ier lintferıung der langen Rückenmuskeln zum Zweck der Erïffnumg des Wirbelkanals zeigt sich, dass del gangränise Zerfall der Rückeunuskelı voin oben beschriebenen Decubitus aus weitgehende, zilm Theil sinü̈se Zerstïrungen der Muskeln, graugrüne Fïrbung und Zerfall derseltien zu weicher schmieriger Masse, da und dort auch reactive Entzündung mit kleinen Eiterdepots herrorgerufen hat.

Die bei der Grïffinung des Wirbelkanals präparirten. resp. durchsäoten Knochentheile, die Arcus vertebrarum verhalten sich normal.

Der blosigelegte Dorsaltheil der Dura mater spinalis zeigt an seiner Aussenfläche kieine Veränderung, erscheint weiss, von normalem Gílanz. Der Durasack ist im Lendenabschuitt stärker gespannt, als im Dorsalabschnitt. Die Flüssigkeit in Arachnoidealraum von normaler Menge und Beschaffenheit.

Das Rückennark zeigt weder im Cervical- noch im Dorsalabschuitte irgend welche Veränderung der Cousistenz und Configuration. Die Pia mater ist blutarm, desoleichen in hohem Haasse das Rückenmark selbst, dessen graue Substanz ron der glänzend weissen durch einen hellgraueren Tou mur schwach unterschieden ist. Die abgehenden Nerrenwureln des Cervicalund Dorsalmarkes sind you normaler Farbe, Consistenz, Dicke.

Auders verhält es sich im Lendenahschnitte. Nan erkennt sofort, dass die Lumbalanschwellung und die Canda equina durch einen längs der

1) Ich kann nicht mmhin, hier darauf binzuweisen, dass die Befolgung der alten hewährten Sectionsregel, wonach die Nieren und IIarnleiter als extraperitoneale Organe erst nach llinwegnahme aller intraperitonealen Organe für sich untersucht werden, im vorliegenden Falle you grossem Nutzen war. Die geschilderten Verhältnisse der Fortsetzung des Nierenkrebses in die Vella renalis und Vena spermatica interna würden bei Befolgung der gegenwärtig ziun strengen Gesetz erhobenen Art des Secirens, wonach die Nieren sofort nach Herausnahme der Mily entfernt werden, woll kaum so rein und eingehend zu studlieren gewesen sein, als dies hei Befolgung der alten Sectionstechnik der Fall war. 
hinteren Fläche der Wirbelkörper verlaufeuden Tumor nach hinten gedrängt sind. Dieser Tumor hat ausserhalb des Duralsackes zwischen dessen vorderer Fläche lnd der hinteren Fläche der Wirbelkörpe $r$ in dem hiel gelegenen Fett-Zellgewebe seine Ausbreitung der Länge nach (reichlich $6 \mathrm{~cm}$. betragend) genommen, und in dieser Ausdehnung die Dura mit dem Rückenmarke nach hinten verdrängt. Die Innenfläche des Iurasackes, insbesondere auch dessen vordere Fläche erscheint normal, von weisser glänzender Farbe.

Den höchsten Grad erreicht die beschriebene Verdrängung der Dura mater nach hinten in der Höhe des I. Lumbalwirbels, wo der von der Dura bedeckte Tumor in Form eines kleinfingerdicken Quer-Wulstes ins Lumen des Wirbelkanales vorspringt. I)ieser Stelle entsprechend ist das untere Ende der Lumbalanschwellung etwa $3 \mathrm{~cm}$ oberhalb des Rückenmarksendes stark zusammengedrückt. Auf der Höhe dieser stärksten Vorwölbung ist die Vorderwand der Dura mater an einer etwa pfefferkorngrossen, kreisrunden Stelle durchbrochen, und durch diese kleine scharfrandige Perforationsöffnung ragt ein linsengrosser, graurother rundlicher Tumor, knopf- oder pilzförmig, frei in den Durasack herein, ohne hier eine weitere Ausdehnumg zu gewinnen.

Die Pia mater der Lendenanschwellung und die Cauda equina erscheint überall durchaus normal, nur etwas stärker injicirt, insbesondere die ge schlängelt verlaufenden Venen stärker gefüllt, als dies an den höher gelegenen Abschnitten des Markes der Fall ist. Auch die aus der Lendenanschwellung ontspringenden Nervenwurzeln der Cauda equina zeigen weder in Farbe noch Consistenz irgend welche Veränderungen. Die Druckstelle des Räckenmarks hat eine Ausdehnung von höchstens Kleinfingerdicke. Sie ist etwas weicher, aber glänzend weiss. Das oberhalb und unterhalb der Knickungsstelle gelegene Rückenmark lässt ausser Anämie weder in Consistenz, noch Collfiguration die geringste Veränderung erkennen.

Es sei hier aus der nachträglichen mikroskopischen Untersuchung Folgendes eingefrigt:

Die nikroskopische Untersuchung der erweichten Stelle des Rückenmarks zeigt, dass man es nur mit einer geringfügigen, durchaus nicht totalen einfachen Druckatrophie ohne alle entzündlichen Veränderungen zu thun hat. Es finden sich neben wohl erhaltenen Elementen feinkörniger Detritus, im Zerfall begriffene, geschrumpfte oder feinkörnig getrübte Ganglienzellen mit unregelmässigen Contouren etc., Trümmer von Nervenfasern mit geronnener Myelinscheide, varicöse und gequollene Axencylinder, sowie Myelinkugeln, dagegen nur ausserordentlich spärliche Köruerzellen, keine Leucocyten, keine Corpora amylacea.

Die Untersuchung der Iumbalen Nervenwurzeln in ihrem Verlaufe durch den Duralsack zeigt, trot' wiederholter Untersuchung diverser Wurzeln keinerlei Anomalien, insbesondere nicht die Zeichen der Degeneration. ,Nach Entfernung des Rückenmarkes und der Dura mater tritt die in dem Fettzellgewebe zwischen hinterer Fläche der Wirbelkörper und dem Durasacke gelegene Krebswucherung zu Tage. Diese erstreckt sich von der Höhe des 11. Brustwirbelkörpers nach abwärts bis zum 3 Lendenwirbel, an beiden Enden sich zuspitzend, in der Mitte die grösste Ausdehnung erreichend. Diese liegt in der Höhe des 1. Lendenwirbels, wo auch die oben beschriebene Compression des Rückenmarks ihren Sitz hat. Die Krebsmasse lässt sich leicht aus dem Wirbelkanale herausnehmen, sie hängt weder mit dem wohlerhaltenen Lig. Iongitud. posterius, welches die Hinterfläche der Wirbelkörper bedeckt, noch mit der Vorderfläche der Dura fester zusammen. Nur an einer Stelle in der Höhe des 1. Lendenwirbels ist das Lig. Iong. posterius in der Ausdehnung etwa eines 20 Pfennigstückes von der Krebsmasse durchbohrt, und von hier aus gelangt man nach vorne vordringend in den krebsigen Körper des 1 . Lendenwirbels.

Nach Herausnahme des Lenden- und unteren Brusttheiles der Wirbelsäule wird diese ihrer Länge nach durch die Mitte der Wirbelkörper aufgesägt. Dabei zeigte sich:

1) ein kirschkerngrosser grauer Krebsknoten im Innern des Körpers des 12. Brustwirbels, überall von normaler Spongiosa umgeben;

2) ein kirschkerngrosser Krebsknoten im 1 Lendenwirbel, welcher sich bis an die hintere Fläche des Wirbels erstreckt, das Lig. Iongitudinale posterius durchsetzt und wie oben beschrieben, in dem Fettzellgewebe zwischen Wirbelkörper und Dura weiter wuchert;

3) ein erbsengrosser Krebsknoten inmitten des 2. Lendenwirbels und

4) ein erbsengrosser Krebsknoten im Innern des 3. Lendenwirbels.

Die Wirbelkörper besitzen ihre normale Gestalt und Höhe, sind luirgends zusammengesunken oder eingeknickt. Von Interesse ist das Verhaltell der Krebswucherung zu den Foramina intervertebralia. Die weichen Krebsmassen umwuchern die aus dem Durasacke aus- und in die Foramina intervetebralia eintretenden Nerven und die daselbst gelegenen Intervertebralganglien aufs allerdichteste. Dagegen lässt sich eine Wucherung des Krebses a u s s e r hal b der Intervertebrallöcher, etwa längs der Plexus, nicht nachweisen. Iie Krebswucherung setzt sich in sämmtliche zwischen 11. Dor'sal- und 4. Lendenwirbel gelegene Intervertebrallöcher hinein fort. Die hier gelegenen Nervenstämme zeigen schon makroskopiscb ein etwas verändertes Aussehen, die Nerven erscheinen hier auf kurze Strecken geröthet und weicher.

Die mikroskopische Untersuchung ergiebt die Zeichen der Neuritis in selten schöner Weise. Das Endoneurium enthält da und dort, besonders reichlich in der Umgebung der Blutgefässe Infiltration mit weissen Blutkörperchen. Auffallend gering sind aber auch hier neben den entzündlichen Veränderungen die Zeichen der Degeneration der Nervenfasern. Die weitaus grösste Mehrzahl der Nervenfasern Iässt keine Verănderungen erkennen. Doch fehlen diese nicht gänzlich, indem die bekannten Bilder der Zerklüftung und scholligen Fragmentirung der Markscheide, der feinkörnigen Trübung der ganzen Faser, der Aufquellung der Axencylinder bis zum körnigen Zerfall derselben, endlich leer zusammengefallene Schwann'sche Scheiden, wenn auch spärlich angetroffen werden. Leider unterblieb die mikroskopische Untersuchung der peripheren Nervenstämme.

Epikrise. Der vorliegende Fall giebt zu einer Reihe interessanter Betraclitungen Anlass. Zunächst die Thatsaclee, dass sich bei einem, voll früherer Syphilis abgesehen, gesunden 24jährigen Mädchen während der Gravidität ein primärer Nierenkrebs entwickelt, der läıgere Zeit hindurch das Allgemeinbefiıden intact lässt, insbesondere keine Krebskachexie herbeiführt. Dass Nierenkrebs nicht selten im jugendlichen AIter, ja sogar im Kindesalter vorkommt, ist eine bekannte Thatsache. Unter allderen 4 Fällen von primärem Nierenkrebs, welche auf der medicinischen Abtheilung des Bürgerhospitales im Laufe der letzten $4 \frac{1}{2}$. Jahre beobachtet wurden, betraf 1 Fall einen 22jährigen kräftigen Maun, ein anderer einen 26 jährigen. Es ist ferner auffallend, dass sämmtliche 5 Fälle die linke Niere betrafen.

Dass primärer Nierenkrebs oft eine sehr lange Daller hat, ging aus einem dieser Fälle (J. Weiss) hervor, wobei eine mindestens 2 Jahre dauernde intermittirende Hämaturie bestand, ehe es zu den Erscheinungen des Kräfteverfalls, zu Metastasen und zur Krebskachexie kam. In sämmtlicheı 5 Fällen war die rechte Niere gesund. In keinem dieser 5 Fälle wurden mit dem Urin morphotische Bestandtheile entleert, welche als krebsige zu erkennen waren. Stets handelte es sich um Fälle von reiner Hämaturie. In einem Falle kam es während des ganzen Verlaufes niemals zur Häınaturie, in einem anderen bestand anfänglich Hämaturie, welche später völlig sistirte und normaler Harnbeschaffenheit Platz nachte. In beiden Fällen klärte die Section den Mangel, resp. das Sistiren der Hämaturie auf, indem in beiden Fällen der Ureter $5-6 \mathrm{~cm}$. unterhalb seines $\mathrm{Ab}$ ganges aus dem Nierenhilus mit der Aussenfläche des Nierentumors fest verwachsen und dadurch vollständig verschlossen war. Die Nierenblutung muss aber auch aufhören oder, wie in unserem Falle, später geringer werden, wenn die Krebsmassen das ganze Nierenbecken und deı Ureter erfüllen, oder wenn das gesammte secernirende Parenchym krebsig entartet ist, wobei die vis a tergo, die Harnabsonderung fehit, welche das Blut durch den Ureter hindurch in die Harnblase spült.

In keinem der angeführten 5 Fälle von primärem Nierenkrebs wurdeı grössere Blutgerinnsel mit dem Harn entleert. Dagegen beobachten wir gegenwärtig einen Fall, wo bei sicherer Diagnose „Nierenkrebs" häufig $6-10 \mathrm{~cm}$. Iange, drehrunde, wurmförmige derbe Blutgerinnsel mit dem Harn entleert werden. Patient ist mittlerweile gestorben und hat die Section die Diagnose Nierenkrebs bestätigt.

Die klinischen Erscheinungen von Seiten des Nervensystems finden in den Resultaten der anatomischen Untersuchung ihre volle Erklärung.

Das Bild, das unsere Kranke in so klassischer Weise darbot, war dasjenige, welches zuerst Cruveilhier mit dem Namen der Paraplegia dolorosa bezeichnete und Charcot in Anbetracht der ganz enormen Schmerzhaftigkeit der paraplegischen Unterextremitäten bei Wirbelkrebs gewissermaassen als für diesen charakteristisch unter dem Namen "Paraplegie douloureuse des cancéreux" hervorhob.

In der That würde die mit enormer Hyperästhesie und Hyperalgesie, die mit den heftigsten continuirlichen Schmerzen einhergehende Paraplegie der Unterextremitäten im Stande gewesen sein, auf Grund des von Charcot bezeichneten Krankheitsbildes blindlings die Diagnose auf Lendenwirbelkrebs zu stellen. Ich sage blindlings, da eine solche Annahme in unserem Falle weder durch den Nachweis irgend eines Krebstumors provocirt wurde, noch auch dadurch wahrscheinlich wurde, dass unsele Patientin, eine 24jährige, gesunde, wohl aussehende Person, im 6. Monat gravida und zudem früher syphilitisch war.

Es schien uns die Annahme einer Neuritis ascendens (siehe oben die Krankengeschichte) oder eines Gumma resp. einer syphilitischen Pachymeningitis weitaus wahrscheinlicher.

Dass unter solchen Umständen auch die Hämaturie, welche ohne jegliche andere Anzeichen von Nierenkrebs (Tumor, Krebszellen im Harn) bestand, zur Diagnose Nierenkrebs nicht bestimmte, wird als diagnostisches Versehen nicht anzurechnen seiul.

Es verlohnt sich auf unserén Fall etwas näher einzugehen mit Bezug auf das Verhältniss einiger klinischen Erscheinungen zu dell anatomischen Resultaten.

Das erste Symptom bei unserer Kranken sind heftige Kreuzschmerzen, welche wohl durch die krebsige Degeneration der Niere und insbesondere durch die Krebsmetastasen in den Lendenwirbelu hervorgerufen wurden.

Dazu treten die Symptome der motorischen Schwäche in delr Unterextremitäten. Um diese Zeit besteht eine wenn auch geringe Herabsetzung der Tastempfindlichkeit der unteren Extremitäten. Während sich die motorische Schwäche in kürzester Zeit zur completen Paraplegie ohne alle motorischen Reizerscheinungen ausbildet, entwickeln sich im sensiblen Gebiet die heftigten Reizerscheinungen, nämlicl ${ }_{1}$ intensive Schmerzen, eine bedentende Hyperästhesie und Hyperalgesie mit der Eigenthümlichkeit, dass alle sensiblen Eindrücke (selbst die durch Betastung hervorgerufenen) schmerzhaft empfunden. 
werden, sodass das feinere Unterscheidungsvermögen für die verschiedenen Reizqualitäten, wie Berühren, Drücken, Kneifelı, Stechen u. s. w. beinahe gänzlich verloren geht.

Da wir uun alle diese Symptome von nichts Anderem herleiten können, als von der Entwickelung des Krebses zwischen Wirbelkörper und dem unverletzten Durasacke, von der Wucherung des Krebses rings um die aus dem Durasacke aus- und in die Intervertebrallöcher eintretenden Nervenwurzeln - denn die Rückenmarkscompression gehört, wie schon die geringfügigen Zeichen der Degeneration beweisen, wohl erst einem späteren Stadium an - so kommen wir nothgedrungen zu dem Resultat, dass die, die gemeinsamen Nervenwurzeln in den Intervertebrallöcherı umwuchernde und daselbst zweifellos auch durch Compression wirkende Neubildung auf die notorischen Fasern der gemiscliten Stämme anders einwirkte, als auf die sensibelı. Wälırend nämlich keinerlei Zeichen von Reizung der motorischen Stämme, keine Contracturen, klonischen Zuckungen, gesteigerten Reflexe vorliegen, sondern voll Anfang an die Zeichen der Lähmung, sind die Symptome von Seiten der seusibeln Nerven die der intensivsten Reizung. Die heftigen spontanen Schnerzen in dell Extremitäten rühren her von der excentrischen Projection der Schmerzempfindung, welche durch die Reizung der sensibeln Nerven in den Intervertebrallöchern hervorgerufen wurde. Die lier gesetzte Veränderung in den sensibeln Nerven ist auch die Ursache, dass jeder von der Peripherie herkommende sensible Strom, gleichgiltig ob er daselbst durch eine Tastbewegung oder durch Druck, Steclıen etc. hervorgerufen wurde, beim Durchgang durch die entzïndete Nervenstrecke in eine Schmerzempfindung umgesetzt wurde. Ich begnüge mich mit dieser aphoristischen Darstellung, da ein tieferes Eingehen auf diese interessante Frage nicht in meiner Absicht liegt.

Beispiele der Art, dass bei Neuritis eines gemischten Nerven die dadurch hervorgerufeneı motorischen und sensibelı Erscheinungen eine auffallende Incongruenz darbieten können, liegen manche vor.

Vor Kurzem hat Rotl (in Basel) einen durch die Nekropsie erlärteten Fall von Neuritis disseminata (Corresp.-Bl. für Schweizer Aerzte 1883, No. 13) mitgetheilt, wobei trotz der durch die Neuritis gemischter Nerven bedingten schweren Lähmungserscheinungen Schmerzen gänzlich fehlten und die sensibelı Erscheinungen sich nur allein auf Parästhesien und Erweiterung der Tastkreise bescliränkten. Herr Prof. Dr. Leichtensterı theilt ınir einen anderı, in dieser Hinsicht interessanten Fall mit, voll eng umschriebener Compression der Cauda equina in Folge eines Traumas (geleilter Wirbelbruch). An der queren Compressionsstelle waren sämmtliche Wurzelı der Cauda untereinander und mit der Dura eng verwachsen, wie mit einem Stricke fest zusammengeschnürt. Es bestand beträchtlicle Herabsetzung der Sensibilität der Unterextremitäten bei wohl erhaltener grober motorischer Kraft, aber schwerer Ataxie, und bei secundärer aufsteigender Degeneration der Goll'schen Stränge.

Auch das selır frülızeitige gänzliche Erlöschen der Sehnen- und Hautreflexe in unserem Fall hat seine Ursache in der Affection der Nervenwurzelı innerhalb der Foramina intervertebralia. Uebrigens ist hierbei auch der tiefe Sitz der Compressionsursache in Betracht zu ziehen, wodurch die reflexvermittelıde Bahn im Lumbaltleile des Rückenmarks selbst in Mitleidensclaft gezogen und so direct functionsunfähig geinacht worden sein kann. Zum Scllluss möclite ich noch auf das eigentlü̈mliche Verhalten der Nervendegeneration linweisen, welche nur allein in den extraduralen, direct von der Neubildung getroffenen Nervenstämmen nachweisbar war, während die im Duralsack verlaufenden vorderen und hinteren Wurzeln weder Degeneration noch entzündliche Veränderungen erkennen liessen.

(Schinss folgt.) 